\title{
Intestinal Stoma Site Hemorrhage
}

National Cancer Institute

\section{Source}

National Cancer Institute. Intestinal Stoma Site Hemorrhage. NCI Thesaurus. Code C78371.

A finding of blood leakage from the intestinal stoma. 